Journal of

Synchrotron

Radiation

ISSN 0909-0495

Editors: A. Kvick, D. M. Mills and T. Ohta

\title{
The small-angle and wide-angle X-ray scattering set-up at beamline BL9 of DELTA
}

Christina Krywka, Christian Sternemann, Michael Paulus, Nadeem Javid, Roland Winter, Ali Al-Sawalmih, Sangbong Yi, Dierk Raabe and Metin Tolan 
Journal of

\section{Synchrotron \\ Radiation}

ISSN 0909-0495

Received 29 June 2006

Accepted 28 February 2007

\section{The small-angle and wide-angle X-ray scattering set-up at beamline BL9 of DELTA}

\author{
Christina Krywka, ${ }^{a}$ Christian Sternemann, ${ }^{a}$ Michael Paulus, ${ }^{a}$ Nadeem Javid, \\ Roland Winter, ${ }^{b}$ Ali Al-Sawalmih, ${ }^{c} \neq$ Sangbong Yi, ${ }^{c, d}$ Dierk Raabe $^{c}$ and \\ Metin Tolan ${ }^{\mathrm{a}}$
}

a Department of Physics, DELTA, University of Dortmund, D-44221 Dortmund, Germany, ${ }^{\mathbf{b}}$ Department of Chemistry, University of Dortmund, D-44221 Dortmund, Germany, 'Max-PlanckInstitut für Eisenforschung, D-40237 Düsseldorf, Germany, and ${ }^{\mathbf{d}}$ TU Clausthal, Institut für Werkstoffkunde und Werkstofftechnik, D-38678 Clausthal-Zellerfeld, Germany.

E-mail: krywka@physik.uni-dortmund.de

The multi-purpose experimental endstation of beamline BL9 at the Dortmund Electron Accelerator (DELTA) is dedicated to diffraction experiments in grazing-incidence geometry, reflectivity and powder diffraction measurements. Moreover, fluorescence analysis and inelastic X-ray scattering experiments can be performed. Recently, a new set-up for small-angle and wide-angle X-ray scattering utilizing detection by means of an image-plate scanner was installed and is described in detail here. First small-angle X-ray scattering experiments on aqueous solutions of lysozyme with different cosolvents and of staphylococcal nuclease are discussed. The application of the set-up for texture analysis is emphasized and a study of the crystallographic texture of natural bionanocomposites, using lobster and crab cuticles as model materials, is presented.

Keywords: SAXS; WAXS; solution scattering; texture analysis; protein; lysozyme; $\mathrm{X}$-ray diffraction.
(C) 2007 International Union of Crystallography Printed in Singapore - all rights reserved the biomolecule, a mechanism which is essential for its biological activity and aggregation processes.

The combination of small-angle and wide-angle X-ray scattering (WAXS) is widely used for studying the crystallographic texture in polycrystalline nanocomposites and consequently is an important tool in materials science (Fratzl, 2002). The information about the crystallographic texture and the preferred orientation of crystals in polycrystalline materials is inherent in the scattered intensity measured by X-ray diffraction (Kocks et al., 1998). In situ non-destructive measurements of these properties, naturally being features of the bulk material and for this reason only partially accessible through surface-sensitive methods, require a scattering technique that is bulk sensitive. New developments have shown that materials science greatly benefits from structure data gathered in life sciences and have exploited it to develop new materials with specific properties, such as stability, tensile strength, fracture behaviour etc. (Vincent, 1990).

The set-up for SAXS and WAXS recently built up at the endstation of beamline BL9 of the Dortmund Electron Accelerator will be discussed within this article along with some examples for applications of SAXS and texture analysis.

The outline of this paper is as follows. In the next section a short overview of the beamline layout and the possible experimental configurations at the endstation of beamline BL9 are presented. In $\$ 3$ the experimental set-up for SAXS 
and WAXS employing an image-plate scanner is described. First measurements performed in small-angle scattering geometry on aqueous solutions of lysozyme and staphylococcal nuclease are presented and discussed in $\S 4$. Studies of the crystallographic texture of $\alpha$-chitin and calcite in bionanocomposites, lobster and crab cuticle, are described and analyzed in $§ 5$. Finally, conclusions and a short outlook are given in $\$ 6$.

\section{Beamline BL9 of DELTA}

The DELTA synchrotron radiation source is located at the University of Dortmund, Germany (Tolan et al., 2003). The storage ring is operated at a maximum energy of $1.5 \mathrm{GeV}$. Typical electron beam currents of $120 \mathrm{~mA}$ and lifetimes of about $10 \mathrm{~h}$ are achieved. A superconducting asymmetric wiggler (SAW), two undulators and several dipole magnets provide synchrotron radiation for experiments. Beamline BL9 is attached to the SAW and covers an energy range between $4 \mathrm{keV}$ and $30 \mathrm{keV}$ with an energy resolution of $\Delta E / E \simeq 10^{-4}$ by means of a Si (311) double-crystal monochromator (Paulus et al., 2005). The experimental endstation of beamline BL9 is equipped with a six-circle diffractometer and a spectrometer in Rowland geometry. Thus experiments focusing on X-ray grazing incidence and powder diffraction, resonant and nonresonant inelastic X-ray scattering, fluorescence analysis and $\mathrm{X}$-ray standing waves can also be accomplished. A detailed overview of such studies performed at BL9 is given by Krywka et al. (2006). With the installation of a MAR345 image-plate scanner and the adaption of sample stages and beam paths, $\mathrm{X}$-ray scattering experiments in small-angle and wide-angle geometry became feasible, as will be described in the following paragraph. The relevant beamline parameters are given in Table 1.
Table 1

Characteristics of the SAXS/WAXS set-up at BL9 of DELTA.

\begin{tabular}{|c|c|}
\hline Energy range (SAXS) & $4-30 \mathrm{keV}(8-30 \mathrm{keV})$ \\
\hline Energy resolution & $\Delta E / E \simeq 10^{-4}$ \\
\hline Monochromator & $\begin{array}{l}\text { Double-crystal monochromator, Si (311), } \\
\text { sagittally focusing second crystal }\end{array}$ \\
\hline $\begin{array}{l}\text { Focused beam size at } \\
\text { detector }\end{array}$ & $1.0 \mathrm{~mm} \times 1.5 \mathrm{~mm}$ (typically) \\
\hline Beam divergence & $0.7 \mathrm{mrad} \times 7.5 \mathrm{mrad}(\mathrm{h} \times \mathrm{v})$ \\
\hline Flux at $10 \mathrm{keV}$ & $\begin{array}{l}10^{7}-10^{8} \text { photons } \mathrm{s}^{-1} \mathrm{~mm}^{-2} \mathrm{~mA}^{-1} \\
\text { (depending on sample-detector distance) }\end{array}$ \\
\hline Sample-detector distance & $450-3300 \mathrm{~mm}$ \\
\hline Measurable $q$ range & $0.007-5.5 \AA^{-1}$ \\
\hline Detector & $\begin{array}{l}\text { MAR345 imaging plate, } 345 \mathrm{~mm}, 100-150 \mu \mathrm{m} \\
\text { pixel size, readout time } \sim 2 \mathrm{~min} \text {, detective } \\
\quad \text { quantum efficiency }>80 \% \text {, dark count }<1 \mathrm{~s}^{-1}\end{array}$ \\
\hline Measuring times & $1-1800$ s (typically) \\
\hline \multirow[t]{2}{*}{ Sample environments } & Temperature: $253-353 \mathrm{~K}$ \\
\hline & Pressure: $1-7000$ bar \\
\hline
\end{tabular}

\section{Experimental set-up for SAXS and WAXS}

A schematic overview of the experimental set-up for twodimensional SAXS at BL9 of DELTA is depicted in Fig. 1. The monochromated X-ray beam can be vertically focused at the detector position by means of the sagittally bent second monochromator crystal and can be collimated using the slit systems S1 and S2, with S3 being a guard slit to reduce slit scattering. In the present state of the beamline, horizontal focusing is not possible. However, a small adjustable mirror of length $15 \mathrm{~cm}$ can be installed optionally if an increase of flux density is desired (Krywka et al., 2006). A fast-shutter and absorber set (XIA PF2S2 and PF4) are used for exposure control and automatic attenuation of the incoming beam.

For reasons of normalization, the intensity of the incoming beam as well as that of the transmitted beam are measured in front of the sample and behind it, respectively. The latter is measured by a detector which records the air scattering of a

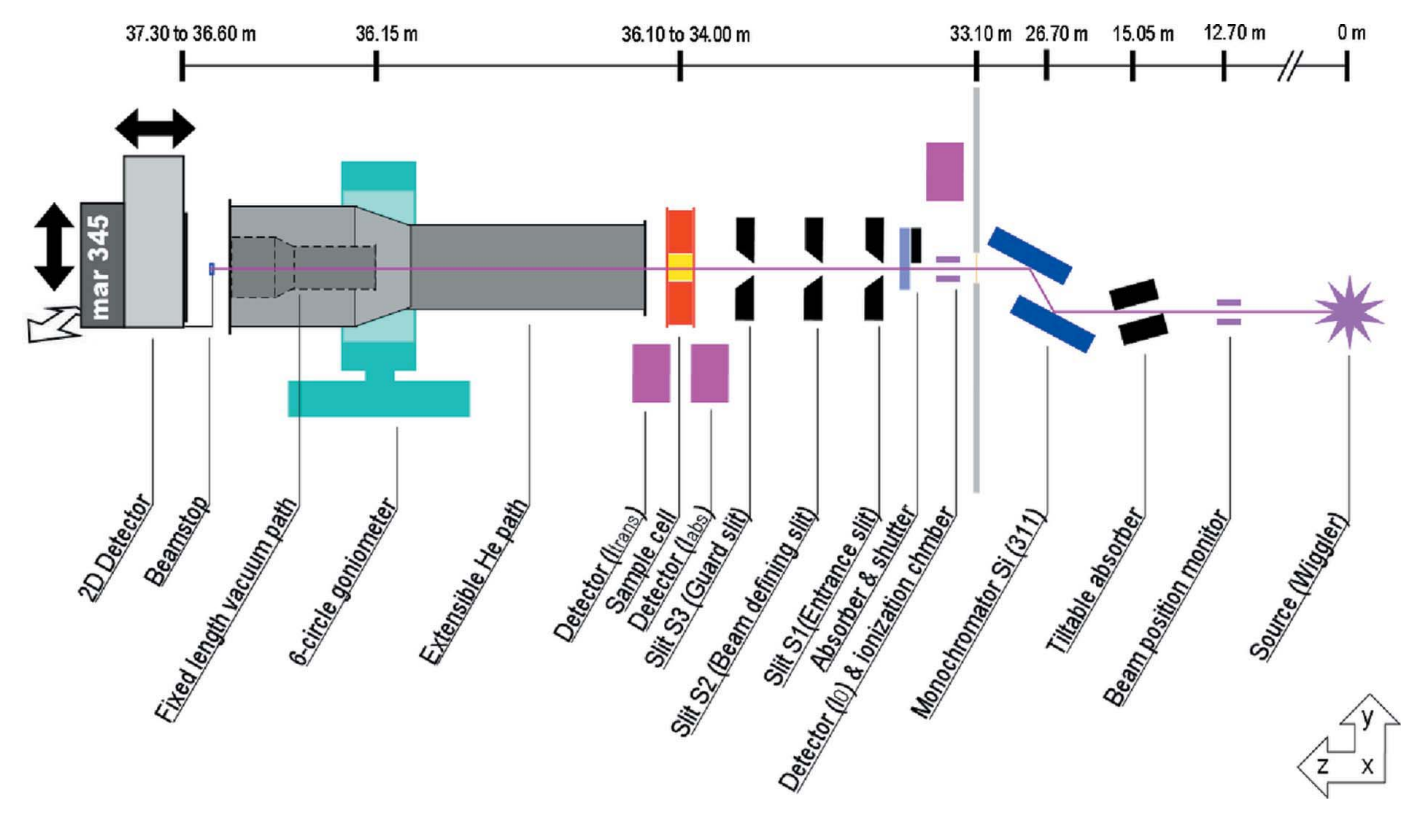

Figure 1

Outline of the SAXS/WAXS set-up on BL9. The sample can be mounted at different sample-to-detector distances, ranging from $0.45 \mathrm{~m}$ to $3.3 \mathrm{~m}$. For the smaller distances the sample can be placed in the diffractometer and the flexible extensible helium path can be reduced in length. 


\section{research papers}

short air path behind the sample. A beam stop with an integrated pin-diode is currently under development so the additional air scattering path can be avoided in future. Along the remaining beam path, parasitic scattering is reduced by employing a modular extensible tube filled with helium and with a large diameter, sufficient for the complete solid angle that can be seen by the detector. Alternatively, an evacuated vacuum tube with a smaller diameter can also be used. The face sides of both tubes are closed with Kapton foils. The helium-filled beam path has proven most useful owing to space restrictions in the experimental hutch and because the experimental hutch of BL9 is frequently used for experiments which require the beam path to be easily unmounted. Unlike the helium beam path, which is suspended on a ceilingmounted rail, the evacuated beam path is significantly heavier and therefore requires a semi-permanent installation. However, for measurements with high resolution at low momentum transfer $(q)$ values, the evacuated beam path is essential.

A lead beamstop of diameter $8 \mathrm{~mm}$ is positioned in front of the MAR detector and held in place by a Kapton foil spread across the active area of the detector. The mounting frame of the beamstop is firmly fixed to the linear translation stage of the detector, thus allowing a positioning accuracy of $12.5 \mu \mathrm{m}$ and $1.5 \mu \mathrm{m}$ in the $x, z$ and $y$ directions, respectively. The total translation path of the detector stage is about $300 \mathrm{~mm}$ in the $x, z$ direction and the sample-to-detector distance can be varied freely within the range $45-330 \mathrm{~cm}$. Detector tilt, detector-to-sample distance and direct beam position can be calibrated by measuring the powder diffraction pattern of a low-angle calibrant sample. The radially integrated diffraction pattern of silver behenate powder, $\mathrm{C}_{21} \mathrm{H}_{43}$ COO.Ag, with a $d$ spacing of $58.376 \AA$, purchased from Rose Chemicals Ltd, UK, measured at an incident photon energy of $11.5 \mathrm{keV}$ and at a sample-to-detector distance of $109 \mathrm{~cm}$, is shown in Fig. 2. Also, the scattering of pure water is shown for both the evacuated and helium-filled beam path set-up. As the scattering of water at low $q$ values only depends on the isothermal compressibility, it has no $q$ dependence and is equal to $1.632 \times$ $10^{-2} \mathrm{~cm}^{-1}$ at $293 \mathrm{~K}$ (Orthaber et al., 2000). The scattering pattern of water can therefore be used for the estimation of the available $q$-range quality of the set-up as well as for normalization of the scattered intensity to an absolute scale.

The samples can be filled into removable easy-to-change containers, which are placed in a sample holder, all of which are shown in detail in the top panel of Fig. 3. Liquid samples can be filled directly into the cavity of the sample container, encircled by a gold-coated copper frame and two MICA windows, each $30 \mu \mathrm{m}$ thick. The containers are available with depths of $1 \mathrm{~mm}$ and $3 \mathrm{~mm}$, and hence require a volume of about 0.1 to $0.2 \mathrm{ml}$, depending on the desired beam path length within the sample. The enclosed sample volume can be temperature controlled in the range $253-353 \mathrm{~K}$. If the volume of the sample needs to be smaller, or in case of a powder-like sample, it can instead be filled into a quartz capillary and subsequently loaded into the sample container. Alternatively, it can also be mounted into a goniometer head. If pressure control is also desired, a high-pressure sample cell is available instead with diamond windows for beam passage $(2 \mathrm{~mm} \times$ $1 \mathrm{~mm})$, shown in the lower panel of Fig. 3. By applying hydrostatic pressure it allows SAXS measurements on aqueous solutions at pressures up to 7 kbar (Malessa, 1998). The liquid sample is then filled into a cavity volume of about $30 \mu$, encircled by Teflon spacers and Kapton foils. Three examples of application of the SAXS/WAXS set-ups are given in $\$ 4$ and $\S 5$.

If the sample has a strong absorption or is a weak scatterer, the signal obtained by the measurements tremendously
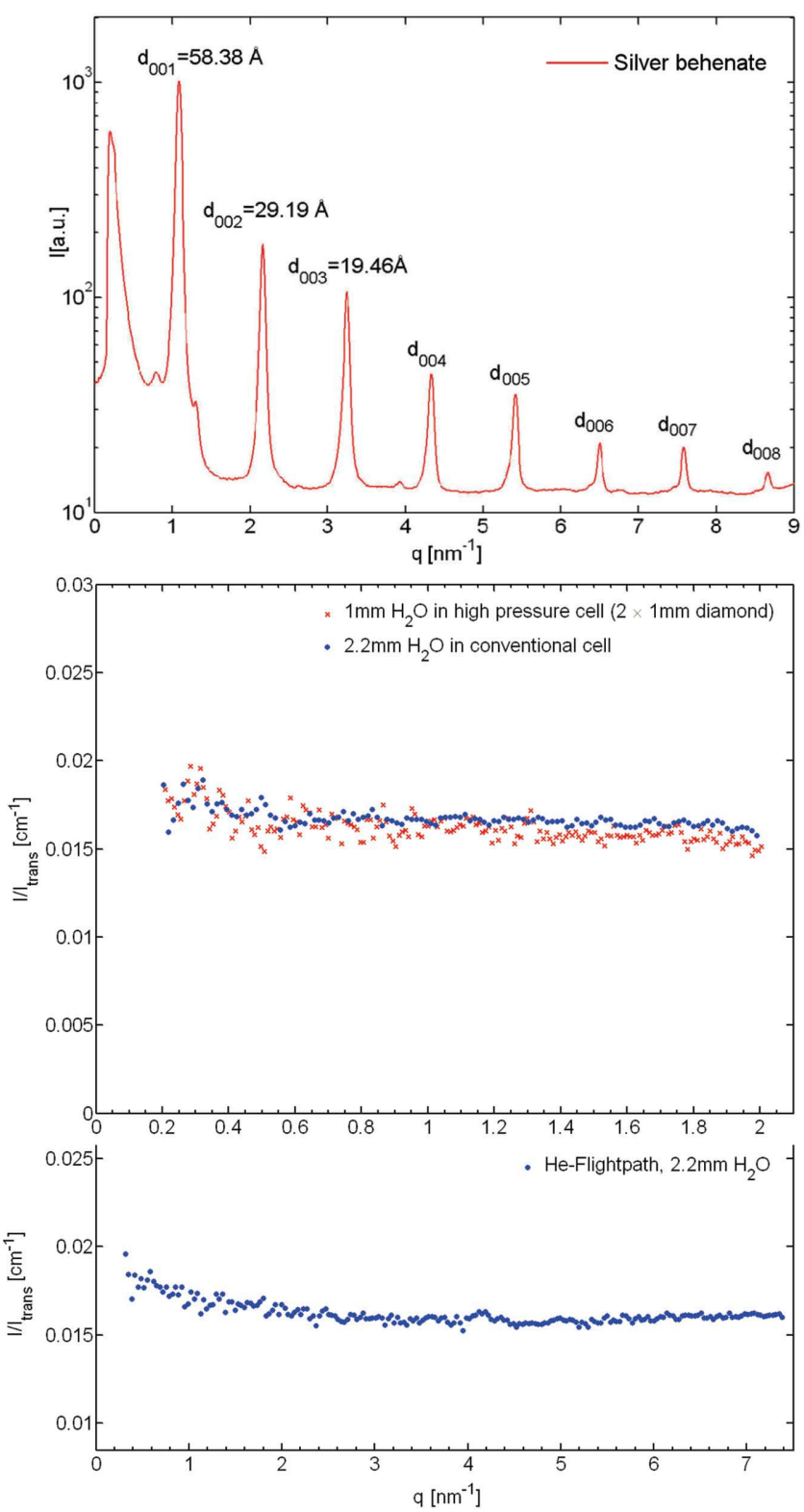

Figure 2

Top panel: radially integrated scattering pattern of a silver behenate calibration sample measured at $11.5 \mathrm{keV}$ incident energy and at ampleto-detector distance of $109 \mathrm{~cm}$ as a function of momentum transfer $q$. Lower panel: scattering pattern of pure water (dots) recorded with both the evacuated and helium-filled beam path (top and bottom, respectively). In addition, a water spectrum measured using the high-pressure sample environment with evacuated beam path is shown (top, crosses). 


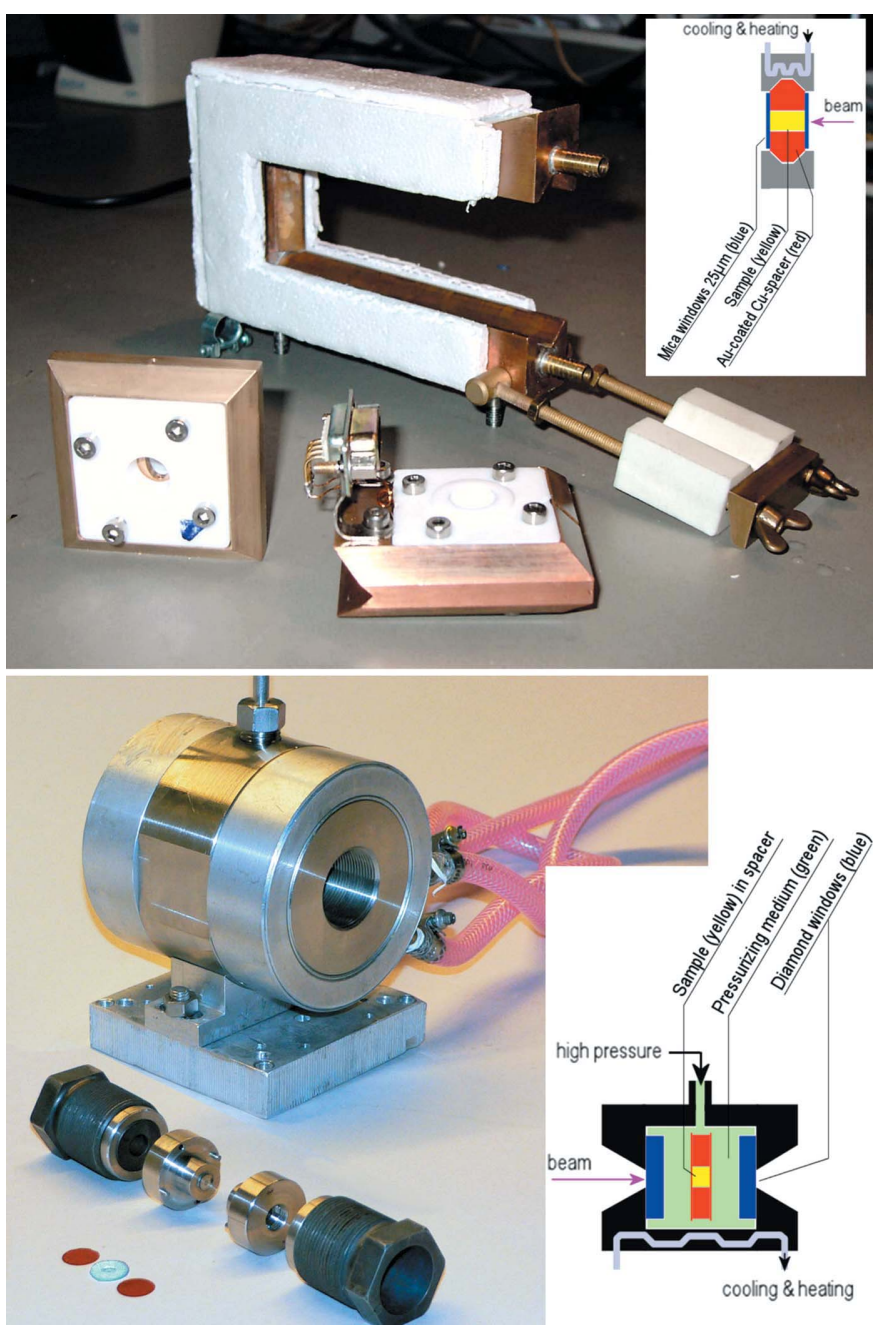

Figure 3

Sample environments for SAXS measurements. The insets show the schematic profile of both sample cells. Upper photograph: the white U-shaped temperature-controlled sample holder can be loaded with an easy-to-change sample container and a temperature-readout sensor (at the bottom of the photograph). Lower photograph: the hydrostatic highpressure cell which allows both temperature and pressure control. At the bottom the diamond window elements as well as the disassembled sample containment are visible.

benefits from the use of a CCD or an image-plate scanner. This is due to the fact that the scattered intensity is recorded in all directions within the solid angle that is covered by the detector. A subsequent radial integration of the scattered intensity, being isotropic in a typical SAXS experiment, leads to a significant improvement in the signal-to-noise ratio of the recorded signal. The detector used at BL9 is a MAR345 imaging plate with a circular active area of diameter $345 \mathrm{~mm}$ and a minimum pixel size of $100 \mu \mathrm{m} \times 100 \mu \mathrm{m}$. Image plates have found great acceptance and widespread use mainly because of their high sensitivity, low intrinsic noise, large dynamic range $\left(\sim 10^{5}\right)$ and relatively long-lived image with half-life times of 1-100 h, depending on the type of the imaging plate (White et al., 1999; Hammersley et al., 1995; Amemiya, 1995). The exposure time of the sample to the $\mathrm{X}$-ray beam can be controlled via beamline software as well as via manual override control of the fast shutter electronics. Mechanical alignment of the detector stage as well as the exposure control are seamlessly integrated into the beamline control software allowing all kinds of scans required to be performed in a SAXS or WAXS experiment. The sample can be mounted either in the diffractometer, providing $x, y, z$ and $\chi, \varphi$ as available translational and rotational degrees of freedom (at small detector-to-sample distance, 45 to $115 \mathrm{~cm}$ ), or on a separate sample stage outside of the diffractometer with only translational degrees of freedom available (at higher detector-to-sample distances). The rotational axes $\chi$ and $\varphi$ are indicated in Fig. 6.

\section{SAXS of aqueous lysozyme and staphylococcal nuclease solutions}

As a part of the human immune system, lysozyme helps to kill bacteria by breaking the carbohydrate chains of the bacteria cell walls leading them to burst under the bacteria's own high internal osmotic pressure. Mucous and tears contain lysozyme to resist infection on exposed surfaces. In the blood, lysozyme also provides some protection along with the more powerful methods provided by the immune system (Goodsell, 2000). Lysozyme contains 129 amino acids and has a molecular mass of about $14.3 \mathrm{kDa}$. It can be extracted from hen egg-white (HEW-lysozyme). The radius of gyration in solution is calculated from the molecular structure (PDB entry 1GXV; Refaee et al., 2003) to be $15 \AA$. The lysozyme used in our experiment was purchased from Sigma Aldrich, Germany.

SAXS images were taken of aqueous solutions of lysozyme at different concentrations of 1, 4 and $10 \% \mathrm{w} / \mathrm{v}$ (weight by volume) in $20 \mathrm{~m} M$ citrate buffer at $\mathrm{pH}$ 4.6. In order to examine the change in tertiary structure of the protein owing to the presence of cosolutes, $\mathrm{NaCl}$, glycerol and trifluoroethanol have been added and measurements were performed at different concentrations. The samples were kept at $253 \mathrm{~K}$ immediately after preparation in order to prevent aggregation and degredation of the lysozyme molecules.

The biological function of staphylococcal nuclease (SNase) is to catalyze the hydrolysis of the phosphate backbone of DNA and RNA. With $16.8 \mathrm{kDa}$ and 149 amino acids it is slightly larger than lysozyme, and the radius of gyration in solution can again be calculated from the given molecular structure (PDB entry 1EY0; Chen et al., 2000) to be $16 \AA$. Unlike lysozyme, this protein can fold and unfold reversibly owing to the lack of disulfide bonds or free sulfhydryl groups. Because of this peculiarity the intermediate states of unfolding next to their native and denaturated states can be studied by applying a reversible condition that induces unfolding or refolding, such as temperature or pressure. As a first approach to the examination of the reversible denaturation process of SNase we have attempted to employ the high-pressure cell at BL9 to measure the SAXS spectra of a low-concentrated aqueous SNase solution $(1 \% w / v)$ in $50 \mathrm{~m} M$ TrisBis buffer at pH 5.5.

Prior to each measurement, the samples were unfreezed and put into an automatic stirring device in order to assure a 


\section{research papers}

homogeneous mixture of the proteins without any aggregation centres. The scattered intensities were accumulated over different time periods, depending on the concentration of the solution, ranging from $900 \mathrm{~s}$ to $1800 \mathrm{~s}$, and being equal to the period during which the sample was exposed to the X-ray beam. In prior measurements no changes in the scattered intensity distribution were found in $1 \%$ solutions of lysozyme that were exposed to the X-ray beam for $900 \mathrm{~s}$ and $1800 \mathrm{~s}$, therefore we assume that no radiation damage and no radiation-induced aggregation need to be considered in the evaluation of the data. To account for the self-decay of the detector-recorded image, the buffer measurement was always measured with the same exposure time. Since the decay of Fuji imaging plates, being used in MAR345 scanners, is only a function of time and does not depend on the exposure level (see Hammersley et al., 1995; Amemiya, 1995), this will compensate for any decay effects in the difference signal that otherwise would become significant owing to long time measurements.

The measured intensities were integrated radially using the program package FIT2D (Hammersley et al., 1994). Furthermore, normalization of the data was required to account for the variation of the intensity of the incoming beam and the absorption owing to the different concentrations of the solutions. Therefore the intensities of the incoming $\left(I_{\text {in }}\right)$ and the transmitted beam $\left(I_{\text {trans }}\right)$ were measured by means of air scattering and accumulated over the experiment time. The normalized intensity is then $I_{\text {norm }}(q)=I(q) / I_{\text {trans }}$. The integrated scattering profile was corrected for background radiation with the scattered intensity of the empty sample cell.

For the lowest concentrated solutions of the pure proteins, where interactions between protein molecules are negligible, the scattered intensities $I(q)$ can be approximated by the particle form factor $I_{\mathrm{P}}(q)$, which is the squared modulus of the spherically averaged Fourier transform of the electron density distribution function $\rho(r)$. This approximation is valid for a solution that is infinitely dissolved. Therefore no interactions between the solved particles can occur, and the scattered intensity is given by

$$
I(q) \simeq I_{\mathrm{P}}(q) \propto\left|\left\langle A_{\mathrm{P}}(q)\right\rangle_{\Omega}\right|^{2}=\int \frac{\sin (q r)}{q r} \rho(\mathbf{r}) \mathrm{d}^{3} r .
$$

The particle form factor has been calculated from the given molecular structure with the help of the program package CRYSOL (Svergun et al., 1995) and was used to find the best fits to the experimental data of lysozyme and SNase.

In the calculation it is assumed that the macromolecule is surrounded by a hydration shell of water with a density different from that of the bulk solvent, as evidenced experimentally by Svergun et al. (1998) and confirmed by molecular dynamics simulations (Merzel \& Smith, 2002). A good agreement with experimental scattering profiles measured on lysozyme solutions could be achieved when a hydration layer with a thickness of $3 \AA$ and an electron density enhanced by up to $15 \%$ compared with bulk water was assumed. With the first hydration layer thickness of $3 \AA$, an increase in electron density of about $4 \%$ (compared with bulk water, 0.334 elec- trons $\AA^{-3}$ ) was calculated from our own experimental data utilizing the package CRYSOL. The deviation from the relative electron density difference predicted by the molecular dynamics simulations might be explained by taking into account the influence of the citrate buffer added to the solution. The buffer can interact with the lysozyme boundary and thus cause a decrease in the electron density contrast.

The background-subtracted scattering profiles of pure lysozyme solutions are shown in Fig. 4, along with the best fit performed using CRYSOL for the $1 \%$ solution. For an assessment of the background, raw buffer and solution scattering profiles from a $1 \%$ solution are also displayed in Fig. 4. The background-subtracted data are in good accordance with previously reported SAXS measurements on lysozyme solutions especially when comparing the position of the onset of the first shoulder of the curve (see, for example, Svergun et al., 1998). The radius of gyration, $R_{\mathrm{G}}=15.2 \AA$, of the native globular protein, which is calculated from the slope of the linear fit in a $\log (I)$ versus $q^{2}$ plot (Guinier \& Fournet, 1955), is also in good accordance with the theoretical value calculated using CRYSOL from the molecular data of $15 \AA$.

A change in the tertiary structure, e.g. induced by an unfolding agent, can be measured by its impact on the radius of gyration which is expected to increase if the conformation of the protein departs from the globular most-compact shape. Measurements were performed on solutions of lysozyme with added cosolutes or cosolvents $\mathrm{NaCl}$, glycerol and trifluoro-

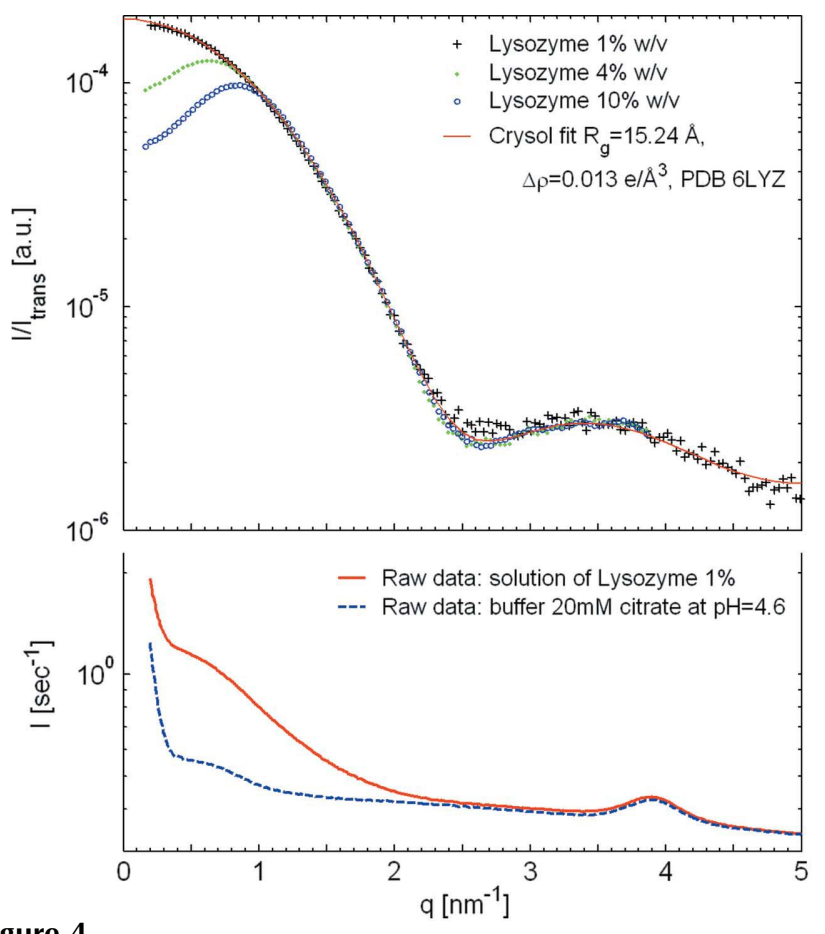

Figure 4

Upper panel: scattered intensities from pure aqueous lysozyme solutions at different concentrations in $20 \mathrm{mM}$ citrate buffer at $\mathrm{pH}$ 4.6. For the $1 \%$ (weight by volume) solution the form factor is calculated from a threedimensional molecular model (PDB entry 1GXV) using the software package CRYSOL and refined to the experimental data. Lower panel: raw (normalized and radially integrated) scattered intensities of $1 \%$ solution and buffer. 
ethanol (TFE). It was shown that a volume fraction of $35 \%$ TFE induced a slight expansion of the molecule owing to a partial unfolding indicated by an increase in $R_{\mathrm{G}}$ of $2 \AA$, whereas it remained unchanged in a solution with a volume fraction of $10 \%$ TFE. The admixture of $\mathrm{NaCl}$, on the other hand, exposed a linear increase in $R_{\mathrm{G}}$, proportional to the added amount of $\mathrm{NaCl}$. The maximum increase in $R_{\mathrm{G}}$ was also about $2 \AA$, at $250 \mathrm{mM}$ added $\mathrm{NaCl}$. As it is not expected for added $\mathrm{NaCl}$ to induce an unfolding of the molecule, the increase in $R_{\mathrm{G}}$ can be explained by either changes in the hydration layer or by association of the protein molecules. Through charge screening the dissociated $\mathrm{NaCl}$ exposes a stabilizing effect on the protein, possibly leading to an increase in the hydration layer thickness. Also, as indicated by previous SAXS and light-scattering experiments on lysozyme and $\beta$ lactoglobulin (Georgalis et al., 1997; Baldini et al., 1999), the increase of the apparent radius of gyration can also be explained by salt-induced association of the monomers into dimers. No change of $R_{\mathrm{G}}$ could be observed in lysozyme solutions with added glycerol. Here the maximum glycerol amount was $2 M$. These results are discussed in more detail by Javid et al. (2007) with the focus on the change of the intramolecular interaction potential of the protein owing to the presence of the cosolvents.

The background-subtracted profile of the SNase solution is shown in Fig. 5. Despite the high absorption of the diamond windows employed in the high-pressure cell (transmission = $20 \%$ at $10 \mathrm{keV}$ ), the measured data expose a good signal-tonoise ratio, and the experimental data could be easily fitted to the form factor calculated from the given three-dimensional molecular model. The radius of gyration was calculated from the experimental data to be $R_{\mathrm{G}}=16.6 \AA$ and is therefore in

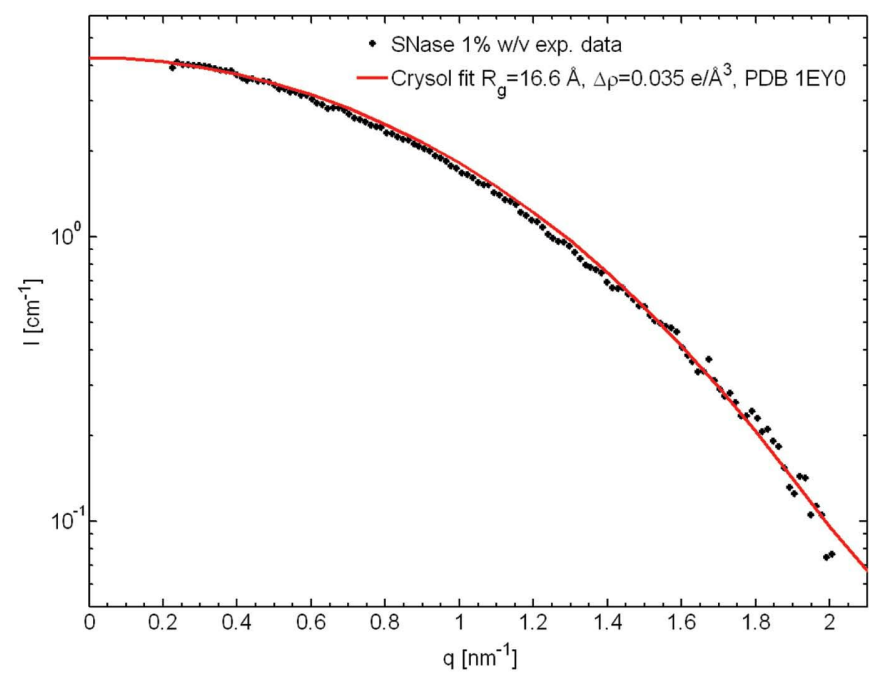

Figure 5

Scattered intensity from pure aqueous SNase solutions at $1 \%(w / v)$ in $50 \mathrm{~m} M$ TrisBis buffer at $\mathrm{pH}$ 5.5. The scattering profile was calibrated to absolute intensity units $\left(\mathrm{cm}^{-1}\right)$ by normalizing the intensity to the previously measured water scattering profile and by dividing it by the volume fraction of the dissolved SNase particles. The form factor is calculated from a three-dimensional molecular model (PDB entry 1EY0) using the software package CRYSOL and refined to the experimental data. good accordance with the theoretical value mentioned above and previously reported values (Panick et al., 1998). As the measurement of water was performed prior to the SNase measurements, the scattered intensity of the SNase solution could be normalized to an absolute scale. The interpolated forward scattering is calculated to be $I(q=0)=4.3 \mathrm{~cm}^{-1}$.

\section{Texture analysis of $a$-chitin and calcite in lobster and crab cuticles}

Crab and lobster cuticles are examples of arthropod exoskeleton that have outstanding mechanical properties, e.g. considerable mechanical strength at very small weight (Vincent, 2002; Ashby et al., 1995). Such biological nanocomposites consist mainly of $\alpha$-chitin fibres, one of the most abundant natural polymers, associated with crystallites of calcite, in a hierarchically designed microstructure of chitin fibres (Raabe, Al-Sawalmih et al., 2005; Raabe, Romano et al., 2005; Raabe et al., 2006). In particular, crab and lobster cuticle are of special interest because the latter contains larger amounts of amorphous calcium carbonate (ACC) than crystalline calcite. The importance of crystallographic texture in biological materials lies in its direct relationship with the possible role of the contained macromolecules in controlling the epitaxy of the minerals during the biomineralization process. Thus the crystallographic orientations of $\alpha$-chitin and calcite were studied in crab and lobster cuticle utilizing wideangle X-ray diffraction by means of two-dimensional detection. All specimens were stored at a temperature of $\sim 243 \mathrm{~K}$ except for the time of the actual synchrotron measurement which in total required about $40 \mathrm{~min}$ to obtain a full set of diffraction patterns for pole figure analysis. The sample geometry with respect to the incident $\mathrm{X}$-ray beam and rotation axis is shown in Fig. 6.

The incident X-ray energy was $15.5 \mathrm{keV}$ in order to minimize sample size effects owing to sample absorption.

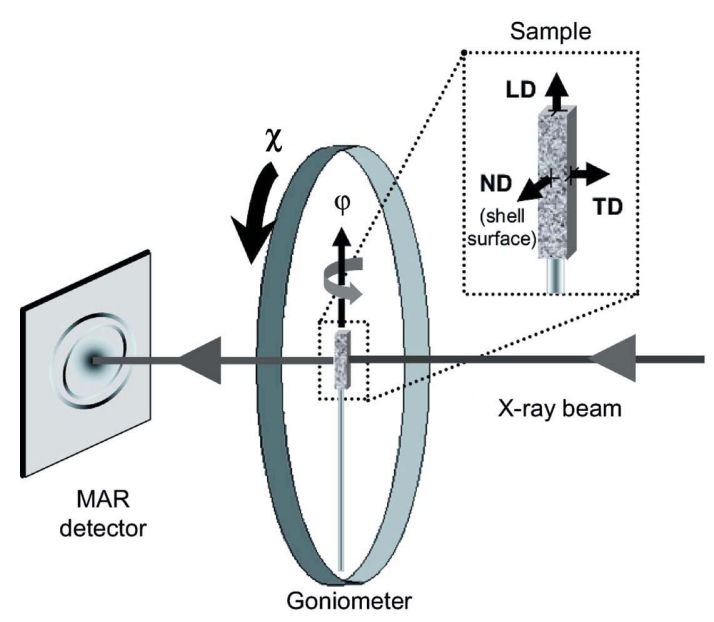

Figure 6

Sample geometry and experimental set-up. $\varphi$ indicates the vertical rotation axis perpendicular to the incident beam to obtain full pole figures. ND, LD and TD refer to normal, longitudinal and transversal axes, respectively. $\chi$ indicates the sample tilt around the axis of the incident beam (fixed during the measurement). 

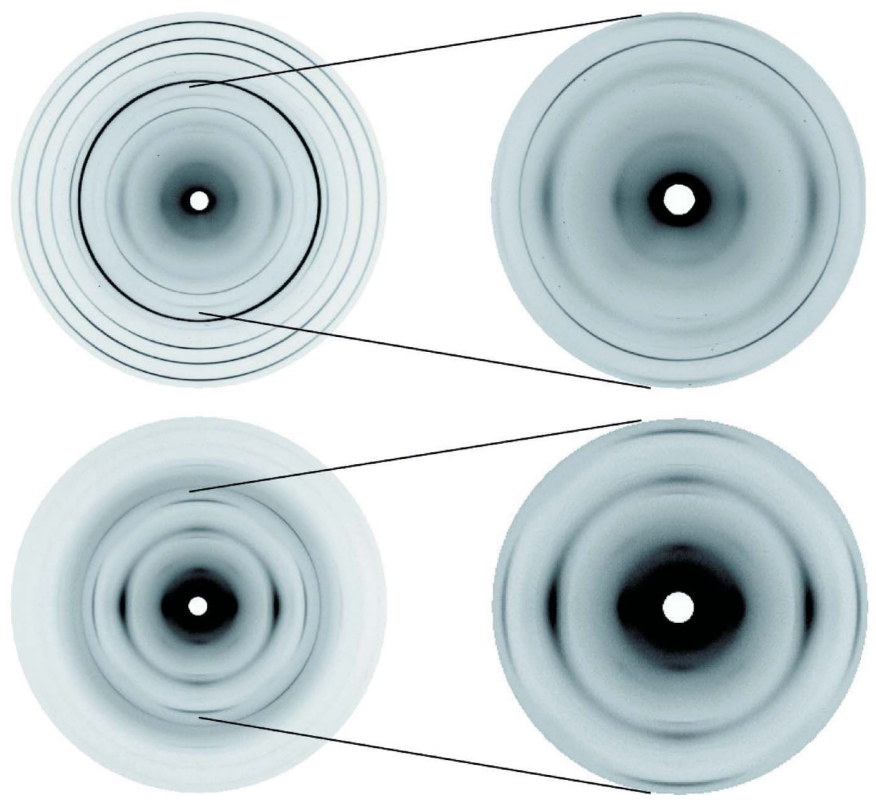

Figure 7

Raw diffraction patterns of crab cuticle (top) and lobster cuticle (bottom) measured using the MAR345 image-plate scanner. They display the circular rings corresponding to the diffraction cones. The total spectrum is shown on the left-hand side and the inner part, representing the $\alpha$-chitin diffraction cones, is highlighted on the right-hand side.
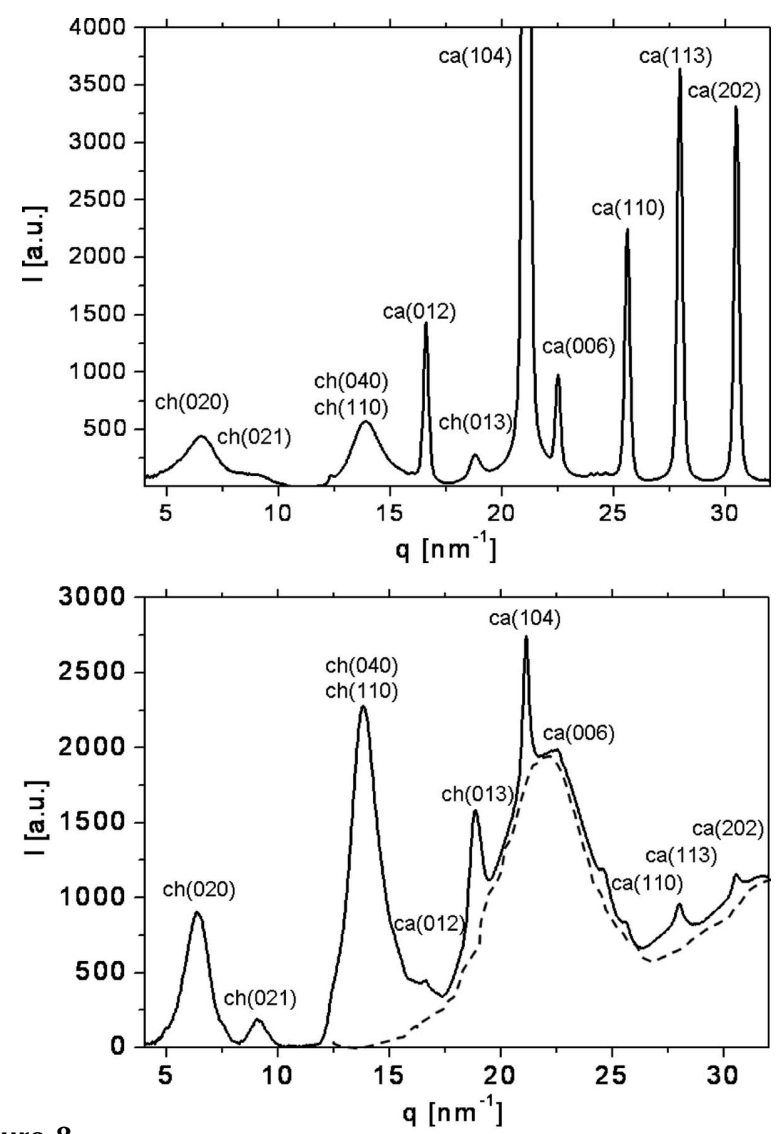

Figure 8

Radially integrated diffraction patterns of crab (upper panel) and lobster cuticle (lower panel) on a $q$ scale. The reflections originating from $\alpha$ chitin (ch) and calcite (ca) are indicated. The broken line refers to the contribution of the amorphous biomineral (ACC) in the lobster cuticle.

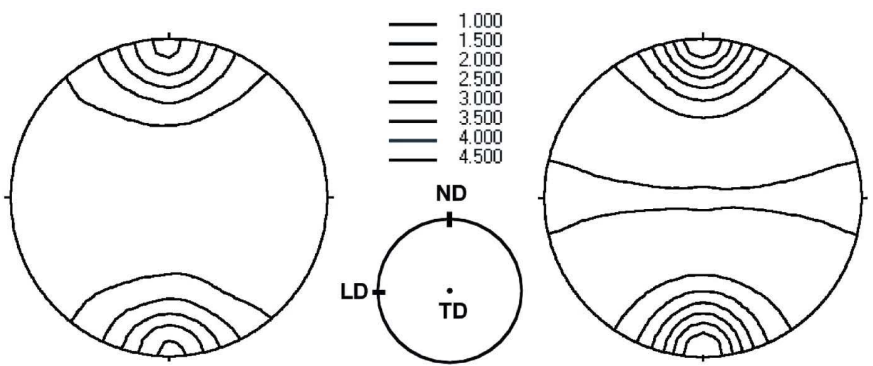

Figure 9

Pole figures of the $\alpha$-chitin $\{020\}$ in crab cuticle (left) and in lobster cuticle (right). The reference pole figure with respect to the sample geometry is also indicated (see Fig. 6 for the ND, LD and TD axes). The scale shows the isolines which represent the texture strength.

Measurements were accomplished using the MAR345 imageplate scanner and carried out at sample-to-detector distances of $380 \mathrm{~mm}$ and $900 \mathrm{~mm}$ and with exposure times between $60 \mathrm{~s}$ and $240 \mathrm{~s}$. MAR images were recorded at different values of $\varphi$ between $0^{\circ}$ and $180^{\circ}$ in $3^{\circ}$ steps. Typical diffraction images of crab and lobster samples are presented in Fig. 7. The diffraction cones corresponding to the calcite are apparent in the outer part of the image (left-hand side). They are much weaker in the case of the lobster owing to the dominating contribution from ACC. The diffraction cones from $\alpha$-chitin, which are highlighted in the magnified area of the MAR images, apparently show a strong texture. The radially integrated spectra are presented in Fig. 8 on a momentum scale and clearly indicate the strong contribution from the amorphous biomineral in lobster cuticle.

The diffraction images were processed using the program package FIT2D (Hammersley et al., 1994). Pole figures were calculated after correction for background and incident intensity decay, employing a program written by S. B. Yi, one of the authors (Yi et al., 2006). The $\{020\}$ pole figure of $\alpha$-chitin shows the direction of the $\alpha$-chitin $b$-axis with respect to the sample geometry given in the reference pole figure. $\alpha$-Chitin $\{020\}$ pole figures for lobster and crab cuticles are presented in Fig. 9. These pole figures reveal a strong texture of $\alpha$-chitin (020), which indicates a strong alignment of the $\alpha$-chitin $b$-axis towards the surface normal. Another component of (020) $\alpha$-chitin is aligned freely within the surface of the lobster cuticle, but the latter is much weaker than the former.

Moreover, it was observed that the $c$-axis of the hexagonal calcite is aligned in the same direction as the $b$-axis of $\alpha$-chitin, both being oriented towards the surface normal. A more detailed analysis of the pole figures will be published elsewhere.

\section{Conclusion and outlook}

A new SAXS and WAXS set-up was successfully installed at BL9 of the DELTA synchrotron source. Small-angle scattering and texture analysis experiments were performed on aqueous solutions of SNase, lysozyme and solutions with added co- 
solutes and cosolvents, e.g. $\mathrm{NaCl}$ and TFE, as well as on samples of crab and lobster cuticle.

The dependence of the lysozyme SAXS spectra could be well understood in terms of a concentration-dependent intermolecular interaction model. The lysozyme SAXS pattern measured at low concentration could be fitted adequately using the program package CRYSOL. The results for thickness and electron density of the hydration shell are in good agreement with literature values. A change in the tertiary structure of the solvated lysozyme molecules induced by the presence of the cosolvents could be measured through the change in the apparent radius of gyration of the protein molecule.

The availability of sample environment conditions is further extended by introducing a high-pressure diamond window cell, which allows SAXS measurements on aqueous solutions at pressure ranges of up to $7 \mathrm{kbar}$. Using this only recently implemented high-pressure cell, a SAXS spectrum of a lowconcentrated solution of the protein staphylococcal nuclease was measured, despite the high absorption of the employed diamond windows.

The texture analysis of crustacean cuticles revealed a strong $\alpha$-chitin (020) texture, normal to the surface, both in crab and lobster cuticle samples, therefore indicating a correlation of the $\alpha$-chitin $b$-axis with the surface normal of the shell. A further preferred orientation parallel to the surface could be observed with respect to the $c$-axis of the hexagonal calcite.

All examples show that high-quality SAXS and WAXS measurements can be accomplished at beamline BL9 of DELTA using the new set-up with detection by means of an image-plate scanner. A configuration of the SAXS set-up in grazing-incidence geometry will be realised to investigate, for example, ordering phenomena of proteins and lipids at interfaces.

CK is supported by the DFG-Forschergruppe 436 (Project 0210111008). AAS would like to thank the German Research Foundation (DFG) under the framework of the Gottfried Wilhelm Leibniz Award for financial support. The authors acknowledge the DELTA machine group for providing synchrotron radiation and technical support.

\section{References}

Amemiya, Y. (1995). J. Synchrotron Rad. 2, 13-21.

Ashby, M. F., Gibson, L. J., Wegst, U. \& Olive, R. (1995). Proc. R. Soc. London Ser. A, 450, 123-140.

Baldini, B., Beretta, S., Chirico, G., Franz, H., Maccioni, E., Mariani, P. \& Spinozzi, F. (1999). Macromolecules, 32, 6128-6138.
Chen, J., Zu, L., Sakon, J. \& Stites, W. E. (2000). J. Mol. Biol. 303, 125130.

Edwards, A. M., Arrowsmith, C. H., Christendat, D., Dharamsi, A., Friesen, J. D., Greenbaltt, J. F. \& Vedadi, M. (2000). Nature Struct. Biol. 7, 970-972.

Fratzl, P. (2002). Fibre Diff. Rev. 10, 31-39.

Georgalis, Y., Umbach, P., Raptis, J. \& Saenger, W. (1997). Acta Cryst. D53, 691-702.

Goodsell, D. S. (2000). RCSB Protein Data Bank.

Guinier, A. \& Fournet, G. (1955). Small Angle Scattering of X-rays. New York: John Wiley and Sons.

Hammersley, A. P., Svensson, S. O. \& Thompson, A. (1994). Nucl. Instrum. Methods Phys. Res. A, 346, 312-321.

Hammersley, A. P., Svensson, S. O., Thompson, A., Graafsma, H. \& Kvick, A. (1995). Rev. Sci. Instrum. 66, 2729-2733.

Javid, N., Vogt, K., Krywka, C., Tolan, M. \& Winter, R. (2007). Chem. Phys. Chem. 8, 679-689.

Kocks, U. F., Tome, C. N. \& Wenk, H.-R. (1998). Texture and Anisotropy. Cambridge University Press.

Krywka, C., Paulus, M., Sternemann, C., Volmer, M., Remhof, A., Nowak, G., Nefedov, A., Pöter, B., Spiegel, M. \& Tolan, M. (2006). J. Synchrotron Rad. 13, 8-13.

Malessa, R. (1998). Dissertation. University of Dortmund, Germany. Merzel, F. \& Smith, J. C. (2002). Proc. Natl. Acad. Sci. USA, 99, 53785383.

Orthaber, D., Bergmann, A. \& Glatter, O. (2000). J. Appl. Cryst. 33, 218-225.

Panick, G., Malessa, R., Winter, R., Rapp, G., Frye, K. J. \& Royer, C. A. (1998). J. Mol. Biol. 275, 389-402.

Paulus, M., Fendt, R., Sternemann, C., Gutt, C., Hövel, H., Volmer, M., Tolan, M. \& Wille, K. (2005). J. Synchrotron Rad. 12, 246-250.

Raabe, D., Al-Sawalmih, A., Romano, P., Sachs, C., Brokmeier, H. G., Yi, S. B., Servos, G. \& Hartwig, H. G. (2005). Mater. Sci. Forum, 495-497, 1665-1674.

Raabe, D., Romano, P., Sachs, C., Al-Sawalmih, A., Brokmeier, H. G., Yi, S. B., Servos, G. \& Hartwig, H. G. (2005). J. Cryst. Growth, 283, $1-7$.

Raabe, D., Romano, P., Sachs, C., Fabritius, H., Al-Sawalmih, A., Yi, S. B., Servos, G. \& Hartwig, H. G. (2006). Mater. Sci. Eng. A, 421, 143-153.

Refaee, M., Tezuka, T., Akasaka, K. \& Williamson, M. (2003). J. Mol. Biol. 327, 857-865.

Svergun, D. I., Barberato, C. \& Koch, M. H. J. (1995). J. Appl. Cryst. 28, 768-773.

Svergun, D. I. \& Koch, M. H. J. (2003). Rep. Prog. Phys. 66, 17351782.

Svergun, D. I., Richard, S., Koch, M. H. J., Sayers, Z., Kurpin, S. \& Zaccai, G. (1998). Proc. Natl. Acad. Sci. USA, 95, 2267-2272.

Tolan, M., Weis T., Wille, K. \& Westphal, C. (2003). Synchrotron Rad. News, 16, 9-11.

Vincent, J. F. V. (1990). Structural Biomaterials. Princeton University Press.

Vincent, J. F. V. (2002). Composites, A33, 1311-1315.

White, M. A., Watowich, S. J. \& Fox, R. O. (1999). J. Appl. Cryst. 32, $65-70$.

Yi, S. B., Davies, C. H. J, Brockmeier, H. G., Bolmaro, R. E., Kainer, K. U. \& Homeyer, J. (2006). Acta Mater. 54, 549-562. 